\title{
Effect of Different Exercise Training Intensities on Musculoskeletal and Neuropathic Pain in Inactive Individuals with Type 2 Diabetes - Preliminary Randomised Controlled Trial
}

Emily R. Cox ${ }^{\mathrm{a}}$, Trishan Gajanand ${ }^{\mathrm{a}}$, Nicola W. Burton ${ }^{\mathrm{b}}$, Jeff S. Coombes ${ }^{\mathrm{a}}$, Brooke K. Coombes $^{\mathrm{c}^{*}}$

aSchool of Human Movement and Nutrition Sciences, The University of Queensland, St Lucia, Queensland, Australia

bSchool of Applied Psychology, Griffith University, Mt Gravatt, Queensland, Australia

${ }^{c}$ School of Allied Health Sciences, Griffith University, Nathan, Queensland, Australia

*Address correspondence and reprint requests to this author at Griffith University, 170 Kessels Road, Nathan, Australia 4111; Tel: +61 75552 7087; E-mail: b.coombes@griffith.edu.au 


\begin{abstract}
Aims: People with type 2 diabetes (T2D) have a greater prevalence of musculoskeletal and neuropathic pain. This exploratory analysis investigated whether exercise of different intensities leads to changes in self-reported musculoskeletal pain or symptoms of diabetic neuropathy in inactive individuals with type 2 diabetes.
\end{abstract}

Methods: Thirty-two inactive adults with T2D (59\% male, mean age 58.7 $\pm 9.1 \mathrm{yrs}$, median $\mathrm{HbA}_{1 \mathrm{c}} 7.8 \%$ ) were randomised to usual care $(\mathrm{CON})$, supervised combined aerobic and resistance moderate-intensity continuous training (C-MICT), or supervised combined highintensity interval training (C-HIIT). At baseline and 8-weeks, musculoskeletal and neuropathic pain were evaluated using a modified Nordic Musculoskeletal Questionnaire and the Neuropathy Total Symptom Score-6 respectively. Quantitative sensory testing was used to determine thermal, mechanical and vibration detection thresholds, as well as pain pressure thresholds. Adverse events were recorded throughout the intervention.

Results: Compared to $\mathrm{CON}$, reduction in musculoskeletal pain intensity was significantly greater for C-HIIT (MD -5.4, 95\% CI [-10.6 to -0.2], $\mathrm{p}=0.04$ ) and non-significantly greater for C-MICT (MD -5.9 [-12.4 to 0.7], $\mathrm{p}=0.08$ ). Changes in neuropathy symptoms were not different between C-HIIT and CON (MD 1.0 [-0.9 to 2.8], p=0.31), or C-MICT and CON (MD 0.2 [-3.1 to 3.6], $\mathrm{p}=0.89$ ). No differences in sensory function were observed between groups. Similar rates of adverse events were seen in both exercise interventions (19 C-HIIT; 17 C-MICT), all but one of which were mild.

Conclusions: Preliminary data suggests 8-weeks of high-intensity combined aerobic and resistance exercise may be safely prescribed for inactive individuals with $\mathrm{T} 2 \mathrm{D}$ and may reduce musculoskeletal pain but not neuropathic symptoms.

Trial Registration: ACTRN12615000475549

Keywords: Diabetic peripheral neuropathy, high-intensity interval training, moderate intensity continuous training, musculoskeletal pain, randomised controlled trial, type 2 diabetes 


\section{Abbreviations}

CON Usual Care

C-HIIT Combined Aerobic and Resistance High-Intensity Interval Training

C-MICT Combined Aerobic and Resistance Moderate Intensity Continuous Training

E4D Exercise for Diabetes Study

HIIT High-Intensity Interval Training

HR Heart Rate

HRpeak Heart Rate Peak

HRR Heart Rate Reserve

MICT Moderate Intensity Continuous Training

NMQ Nordic Musculoskeletal Questionnaire

NTSS-6 Neuropathy Total Symptom Score

PPT Pain Pressure Threshold

RPE Rating of Perceived Exertion 


\section{$1.1 \quad$ Introduction}

The benefits of physical exercise for the prevention and management of type 2 diabetes are well established, including improved glucose control, cardiorespiratory fitness, muscular strength, muscle mass, and body composition [1]. In contrast, there is little knowledge on the specific effects of exercise training on chronic pain in this population [2]. This is despite growing evidence that people with type 2 diabetes are more likely to develop a range of rheumatological and musculoskeletal problems, and experience both widespread and neuropathic pain [3-5]. Chronic pain is frequently associated with physical, psychological and social issues that may interfere with physical activity participation and overall quality of life [6]. Empirical knowledge regarding the effects of exercise on pain is vital to developing strategies to improve adherence to exercise [7].

Traditionally, exercise interventions for people with type 2 diabetes have examined the effect of moderate intensity continuous training (MICT). However, research has shown that highintensity interval training (HIIT), which involves vigorous exercise interspersed with periods of lower intensity exercise or rest, is superior in terms of improving cardiorespiratory fitness and glycaemic control in people with type 2 diabetes $[8,9]$, and is perceived as being more enjoyable [10]. Previous investigations have not included HIIT in chronic pain programs. It has been suggested that the analgesic effects of exercise are intensity-dependent [11], with higher intensities eliciting greater reductions in pain due to the increased release of endogenous opioids [12]. Due to the time-efficient nature, HIIT may also be tolerable for individuals who are unable to exercise for extended periods. Alternatively, the higher workload of HIIT may cause greater strain on the musculoskeletal system. Acute increases in physical loading, such as when inactive individuals commence an exercise regime, may pose a risk of acute injury or exacerbation of pre-existing symptoms. Estimates suggest one in five people with type 2 diabetes assigned to an exercise and lifestyle intervention report pain or discomfort resulting in an inability to exercise for seven or more consecutive days [13], and one in two report musculoskeletal problems as the reason for not participating in exercise long-term [14].

A combination of aerobic and resistance training is recommended by Exercise and Sports Science Australia [15] and the American College of Sports Medicine and the American Diabetes Association [16] for people with type 2 diabetes. However, exercise studies have typically focussed on aerobic-only training, despite the benefits resistance training can provide to this population $[17,18]$. Furthermore, studies have seldom combined aerobic HIIT with resistance training [19-21]. Therefore, prior to its clinical uptake, it is important to evaluate whether combined aerobic and resistance exercise interventions of different intensities can be effectively and safely applied in people with type 2 diabetes. The aims of the current preliminary study were to evaluate the safety and efficacy of low-volume combined aerobic and resistance HIIT (C-HIIT) and combined aerobic and resistance MICT (C-MICT) versus usual care $(\mathrm{CON})$ in inactive people with type 2 diabetes. 


\subsection{Methods}

The following study was undertaken in a subset of participants from an RCT (Exercise for Diabetes; E4D study) investigating the impact of C-HIIT and C-MICT compared to CON on glycaemic control in people with type 2 diabetes (ACTRN12615000475549). Participants underwent all testing at baseline (before randomisation to either CON, C-HIIT or C-MICT) and after 8-weeks at the University of Queensland, St Lucia. Randomisation occurred in blocks of six (identical allocation ratio) and was stratified based on age (18-55 years and 56-80 years) and sex. An individual not associated with the study completed randomisation and allocation via email. No power analysis was completed for this study because it was an exploratory preliminary study and the sample size was based on recruitment within the period December 2016 to November 2017. Written informed consent was received from all participants. Ethical approval was received from The University of Queensland Medical Research Ethics Committee (Approval \#2015000164) and all investigations were carried out in accordance with the Declaration of Helsinki. This study was registered with the Australian New Zealand Clinical Trials Registry (ACTRN12615000475549).

\subsection{Participants}

Adults aged 18-80 years from South East Queensland, Australia were recruited via the study website (www.exercise4diabetes.webs.com), social media, and advertisements within the local university and Diabetes Queensland newsletters. Potential participants were included in the study if they had medically diagnosed type 2 diabetes with a $\mathrm{HbA}_{1 \mathrm{c}}$ level of $\geq 6.0 \%$ or a fasting glucose level of $\geq 7.0 \mathrm{mmol} / \mathrm{L}$ at baseline testing. Exclusion criteria were as per the American College of Sports Medicine's absolute contraindications to exercise [22] including unstable angina, recent myocardial infarction, coronary artery disease and uncontrolled, symptomatic heart failure. Potential participants were also excluded if they participated in more than 150 minutes of moderate physical activity or 75 minutes of vigorous physical activity per week. The presence of musculoskeletal symptoms and/or chronic pain did not result in exclusion unless there was a specific contraindication. Once assessed for inclusion, participants were invited to participate in this current, optional sub-study.

\section{$2.3 \quad$ Outcome Measures}

\subsubsection{Musculoskeletal Symptoms}

The Nordic Musculoskeletal questionnaire (NMQ) was used to capture the prevalence of ache, pain or discomfort in nine body areas (neck, shoulders, upper back, elbows, wrists/hands, low back, hips/thighs, knees, and ankles/feet) during the preceding year and the preceding week [23]. It also asked whether their symptoms had an impact upon normal daily activities or required healthcare treatments in the preceding year. Responses were given as a yes or no (left and right side of the body were not scored separately). The NMQ has been validated for use in the general population [24]. In addition to the above standardised questionnaire, participants were asked to rate their worst level of pain experienced in each of the nine body areas during the last seven days on an 11-point numerical rating scale, with endpoints 0 (no pain) and 10 (worst imaginable pain). Pain intensity scores across the nine body areas were summed to give 
a total score ranging from 0 to 90 , with higher scores indicating more widespread and severe musculoskeletal pain. An investigator unaware of the group allocation administered the questionnaire.

\subsubsection{Neuropathic Symptoms}

Participants were asked to complete the 6-item Neuropathy Total Symptom Scale (NTSS-6). This questionnaire is used to evaluate the most prominent neuropathy sensory symptoms in people with diabetes mellitus and diabetic peripheral neuropathy, and has been shown to be reliable and sensitive [25]. Participants rated the intensity and frequency of both painful (burning, aching, shooting pain) and non-painful (numbness, prickling and/or tingling) symptoms experienced in the feet or lower limbs in the previous 24 hours. Total scores for the NTSS-6 range from 0 to 21.96, with a score greater than zero indicating the presence of at least one sensory symptom. Clinically significant symptoms are defined as a total score greater than 6 [25]. An investigator unaware of the group allocation scored questionnaire responses.

\subsubsection{Quantitative Sensory Testing}

Quantitative sensory testing included the detection of thermal, mechanical and vibration thresholds, and pressure pain thresholds measured bilaterally over the calcaneum at the insertion of the Achilles tendon. To establish warm/cold detection thresholds, a TSAII Neurosensory Analyser (Medoc, North Carolina, United States of America) applied a ramped stimulus $\left(1{ }^{\circ} \mathrm{C} / \mathrm{s}\right)$ from a baseline temperature of $32^{\circ} \mathrm{C}$. The test was ceased with the participant's first perception of warmth or cool. Minimal and maximal temperatures of $0^{\circ} \mathrm{C}$ and $50^{\circ} \mathrm{C}$ were set. Mechanical detection thresholds were tested using von Frey monofilaments $(008 \mathrm{~g}$ to $300 \mathrm{~g}$ ) applying a series of increasing and decreasing intensities to determine the tactile detection threshold as a geometric average. The participant communicated every time they perceived a light touch. Vibration disappearance threshold was performed with a tuning fork $(128 \mathrm{~Hz}, 8 / 8$ scale $)$ and the participant communicated when the perceived vibration sensation disappeared. Pressure pain thresholds were assessed with a pressure algometer (Somedic, Sösdala, Sweden) with probe size of $1 \mathrm{~cm}^{2}$ and rate of pressure increase of $30 \mathrm{kPa} / \mathrm{s}$. The test was ceased when the participant pressed a button to indicate their first perception of pain. Three repetitions separated by 30 seconds rest were completed for each sensory test, and the mean value recorded.

\subsubsection{Adverse Events}

Participants were advised to report to the study research team any adverse events that occurred during the study period and were questioned about adverse events by the study staff member at each training session. When necessary, the type, incidence, and severity of adverse events (Grade 1: Mild; Grade 2: Moderate; Grade 3: Severe; Grade 4: Life-threatening; and Grade 5: Death) were determined based on the Common Terminology Criteria for Adverse Events (Version 5.0) [26].

\subsubsection{Anthropometry}

Height $(\mathrm{cm})$ and weight $(\mathrm{kg})$ were measured at baseline to calculate BMI $\left(\mathrm{kg} / \mathrm{m}^{2}\right)$. 


\subsubsection{Physical Activity Levels}

The interviewer-guided, long form of the International Physical Activity Questionnaire (IPAQ) was used to quantify self-reported physical activity levels at baseline. The IPAQ is a valid and reliable measure of self-reported physical activity [27, 28].

\subsubsection{Bloods}

After an overnight fast ( $\geq 12$ hours), as well as 48 hours with no exercise, and 24 hours with no caffeine, alcohol, tobacco, and other stimulants, a venous blood sample was taken, according to standard phlebotomy procedures, to determine $\mathrm{HbA}_{1 \mathrm{c}}$ and fasting blood glucose at baseline. Detailed methods can be found in the E4D Study protocol (ACTRN12615000475549).

\subsection{Interventions}

\subsubsection{Exercise Interventions}

An 8-week exercise program with both aerobic and resistance components was prescribed under guidance from an Accredited Exercise Physiologist (AEP). During the supervised sessions, workload, number of repetitions during resistance training, heart rate (HR) and rating of perceived exertion (RPE; Borg 6-20 scale [29]) were recorded by the AEP. The types of resistance-based exercises were the same for both groups and involved a combination of machine-based, bodyweight, and free-weight exercises. The order in which the exercises were performed were: 1) leg press, 2) chest press, 3) leg press repeat, 4) seated row, 5) calf raises, 6) shoulder press, 7) abdominal crunch, and 8) bicep curls.

The C-MICT group trained four times per week - two sessions incorporating both aerobic and resistance training, and two sessions involving aerobic training only. For the two combination sessions, participants completed 22 minutes and 30 seconds of aerobic exercise at $55-69 \%$ of HR peak (HRpeak) followed by 30 minutes of resistance-based exercises at a moderate intensity (RPE 11-13; fairly light to somewhat hard). For the two aerobic-only sessions, participants completed 52 minutes and 30 seconds of aerobic exercise at 55-69\% HRpeak. The study was designed so that the C-MICT group completed a total of 210 minutes of exercise per week, which is consistent with the current exercise recommendations by Exercise and Sports Science Australia for people with type 2 diabetes [15].

C-HIIT trained three times per week with each session incorporating a warm-up (and cool down) for 3 minutes at 50-60\% HRpeak, followed by 4 minutes of high-intensity aerobic exercise at $85-95 \%$ HRpeak and $8 \times 1$-minute intervals of high-intensity resistance exercise at an RPE of $\geq 17$ (very hard). One minute of rest separated each interval. The C-HIIT group exercised for 78 minutes per week, around one third of the time of C-MICT.

\subsubsection{Usual Care}

Participants who were randomised to CON continued usual care for 8-weeks before follow-up testing. They were instructed not to change their physical activity or dietary habits during this period. 


\subsection{Exercise Adherence and Compliance}

Adherence was defined based on attendance at exercise sessions. Compliance was determined from exercise intensities achieved during the exercise sessions. For C-HIIT, the participant's heart rate needed to be in the prescribed aerobic intensity zone during the final 2 minutes of the aerobic interval. For C-MICT, compliance was based on their average heart rate for the aerobic component. For the resistance exercises, self-reported RPE was used to assess compliance.

\subsection{Statistical Analysis}

Baseline descriptive statistics (mean $\pm \mathrm{SD}$ or median[interquartile range]) were calculated and compared between groups using a one-way ANOVA or $\chi^{2}$ tests. Baseline characteristics were compared between participants completing the exercise training interventions or CON with those lost to follow-up using unpaired t-tests. Adherence to the exercise sessions, compliance to the prescribed intensity, and the frequency of adverse events were compared between the two exercise training groups using unpaired t-tests. Changes in outcome measures from baseline to post-intervention were computed and individual data graphically illustrated. Normal distribution was confirmed through the Shapiro-Wilk test. For pressure pain threshold, normality was achieved with exclusion of a single outlier. Generalised linear models, adjusted for baseline, were used to evaluate the effect of exercise training on each outcome. C-HIIT and C-MICT were each separately compared to CON. A third model compared participants randomised to C-HIIT or C-MICT (pooled training intervention) with CON. Data was analysed by intention to treat using Stata 13.0 (StataCorp, Texas) and the significance level set at $\leq 0.05$ for all statistical analyses. A sensitivity analysis was performed excluding participants who did not meet the prescribed aerobic or resistance intensities for at least $80 \%$ of the sessions.

\subsection{Results}

\subsubsection{Enrolment and Baseline Characteristics}

Forty-five participants consecutively enrolled in the E4D trial were invited to participate in the current study. Thirty-two participants consented to participate and were randomised to CON $(n=12)$, C-MICT $(n=10)$ or C-HIIT $(n=10)$ (Figure 1$)$. 


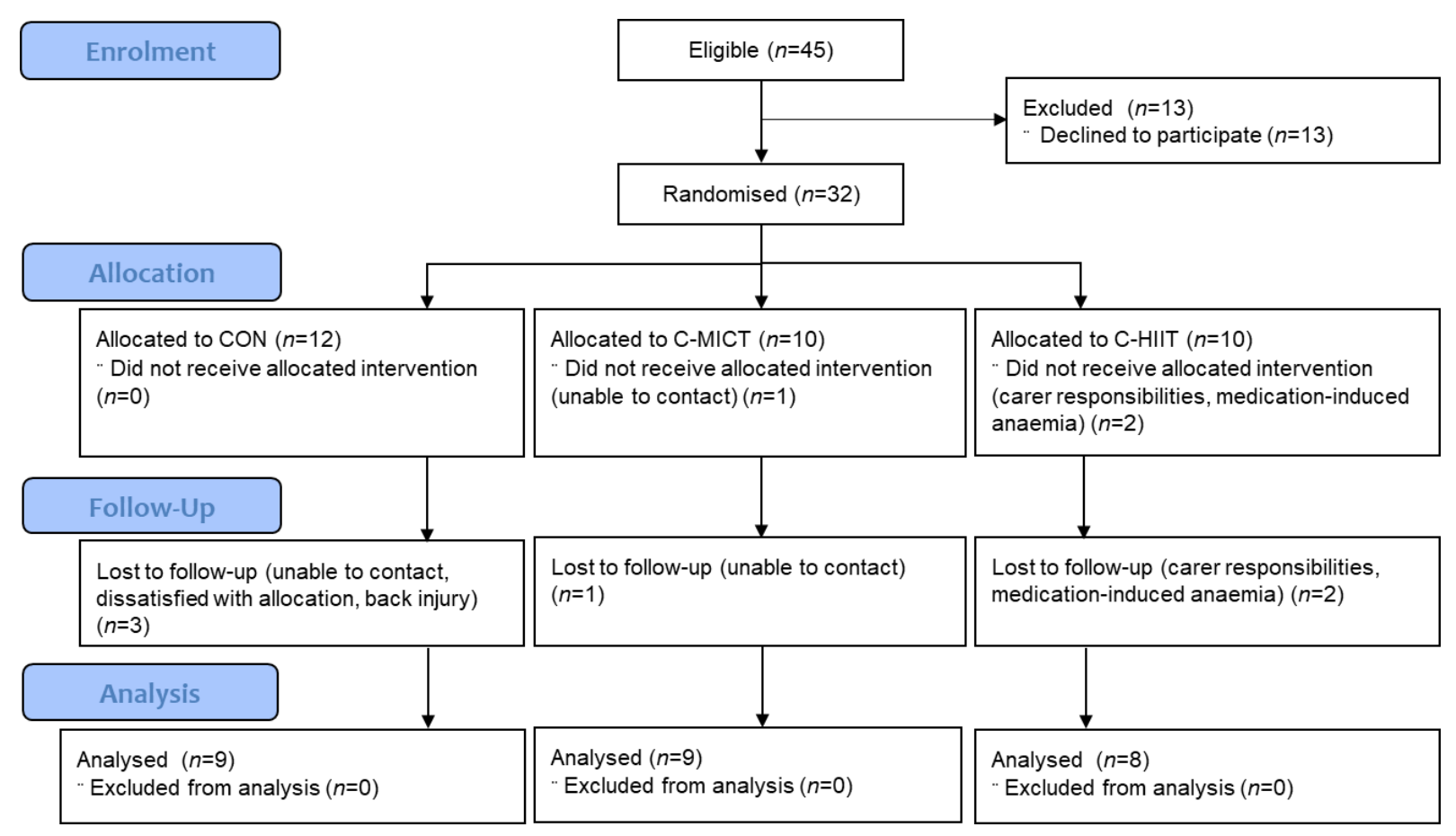

Figure 1: Participant flow following randomisation

Baseline demographic characteristics are described in Table 1. One quarter of participants were classified as obese class 1 (moderate), $28 \%$ as class 2 (severe) and $16 \%$ as class 3 (very severe). Musculoskeletal and neuropathy symptom scores did not differ between the groups at baseline. During the last year, $88 \%$ of participants reported trouble in one or more body regions and $44 \%$ reported being prevented from carrying out normal activities because of this trouble. During the last week, $72 \%$ of participants reported trouble in one or more body regions and $44 \%$ in 3 or more regions. 
Table 1: Baseline demographic and symptom characteristics of randomised participants

$n$

Female sex, $n(\%)$

Age, years

BMI, $\mathrm{kg} / \mathrm{m}^{2}$

Physical activity, MET-mins/week

$\mathrm{HbA}_{1 \mathrm{c}}, \%$

Fasting blood glucose, $\mathrm{mmol} / \mathrm{L}$

Years of diabetes

\section{Musculoskeletal symptoms (NMQ)}

Trouble $^{\mathrm{a}}$ in at least one area in last week

Trouble $^{\mathrm{a}}$ in 3 or more areas in last week

Pain intensity (sum of 9 areas $^{\text {b) }}$ )

Neuropathy Total Symptom Score

(NTSS-6)

Clinically significant neuropathy, $n(\%)^{\mathrm{c}}$

\section{All groups}

32

$13(41 \%)$

$58.7 \pm 9.1$

$34.1 \pm 6.7$

$190[60,270]$

$7.8[7.1,8.9]$

$8.0[6.7,9.4]$

$7[3,15]$

\begin{abstract}
$23(72 \%)$
$14(44 \%)$

$6[0,16]$
\end{abstract}

$2.3[0,7.33]$

$10(31 \%)$

\begin{tabular}{ll} 
C-HIIT & $\begin{array}{l}\text { Between group } \\
\text { p-value }\end{array}$ \\
\hline 10 & - \\
$3(30 \%)$ & 0.66
\end{tabular}

CON
12
$5(42 \%)$
$59.5 \pm 11.1$
$33.5 \pm 6.8$
$191[180,220]$
$7.7[7.4,9.0]$
$8.4[7.0,9.4]$
$8[4.5,19.5]$

\section{0}

$5(50 \%)$

$57.8 \pm 6.9$

$58.7 \pm 9.2$

0.91

$34.7 \pm 7.9$

$34.5 \pm 5.9$

0.69

$225[150,415] \quad 135[40,240] \quad 0.26$

$8.7[7.3,9.0]$

$7.4[7.0,8.2]$

0.26

$7.7[6.7,10.4] \quad 8.3[6.9,8.8]$

$11.5[2,14]$

$5[3,9]$

0.81

0.52

$9(75 \%) \quad 8(80 \%) \quad 6(60 \%) \quad 0.58$

$7(58 \%)$

$5(50 \%)$

$2(20 \%)$

0.18

$5[0,12]$

$8.5[0,17]$

$5[0,16]$

0.50

$2.3[0,7.8]$

$2.7[0,7.3]$

$2.3[1.3,5.3]$

0.74

$3(25 \%)$

$4(40 \%)$

$3(30 \%)$

0.61

${ }^{a}$ Trouble was defined as ache, pain or discomfort at nine body areas as measured by the Nordic Musculoskeletal Questionnaire; ${ }^{b}$ Pain intensity as measured by 11-point numerical rating scale and summed for nine body regions; ${ }^{\mathrm{C} T S S}-6$ scores of 6 or greater were defined as clinically significant neuropathy. 


\subsubsection{Participant Flow}

Twenty-six participants (81\%) completed the study. Of the six participants who did not complete the study, three were from $\mathrm{CON}$ and three from the exercise training groups (one from C-MICT, two from C-HIIT). Reasons for loss to follow-up are provided in Figure 1. Although unrelated reasons were given, baseline neuropathy symptom scores were significantly $(\mathrm{p}<0.001)$ higher for participants who dropped out of the exercise training interventions $(15.4 \pm 6.0)$, than those who completed them (2.9 \pm 2.8$)$. In comparison, there were no differences $(\mathrm{p}=0.15)$ in NTSS- 6 scores for those that dropped out of CON $(0.33 \pm 0.58)$ compared to those that completed it $(5.7 \pm 5.7)$.

\subsubsection{Exercise Adherence}

Participants allocated to C-MICT completed $96.5 \%$ of training sessions and C-HIIT completed $97.9 \%$ of sessions over the 8 -week intervention. Compliance (\%) to the prescribed intensity was $86.6 \%$ for C-MICT and $85.3 \%$ for HIIT. There were no differences in adherence between the training groups ( $>0.05$ ). Four participants (two C-MICT, two C-HIIT) did not meet the prescribed intensity during at least $80 \%$ of sessions.

\subsubsection{Effect of Exercise on Musculoskeletal and Neuropathic Pain}

Changes in pain intensity across nine regions from baseline to post-intervention for individual participants (and mean scores) from each group are illustrated in Figure 2a. Participants assigned to C-HIIT had a greater reduction in pain intensity than those allocated to CON (MD $-5.4,95 \% \mathrm{CI}-10.6$ to $-0.2, \mathrm{p}=0.041)$. Reduction in pain intensity was not significantly different for participants allocated to C-MICT compared to CON (MD -5.9, 95\% CI -12.4 to 0.7, $\mathrm{p}=0.078$ ). Pooling of the two exercise interventions showed a significantly greater reduction in pain intensity compared to CON (MD $-5.1,95 \% \mathrm{CI}-10.2$ to $-0.05, \mathrm{p}=0.048$ ). When participants not meeting the prescribed intensity for $80 \%$ of sessions were excluded, both exercise groups showed a significantly greater reduction in pain intensity compared to CON (C-HIIT vs CON MD -6.9, 95\% CI -12.5 to -1.2, $\mathrm{p}=0.018$; C-MICT vs CON MD $-9.2,95 \% \mathrm{CI}-15.7$ to -0.7 , $\mathrm{p}=0.033$; Pooled exercise vs CON MD $-6.9,95 \% \mathrm{CI}-12.5$ to $-1.3, \mathrm{p}=0.016$ ). 


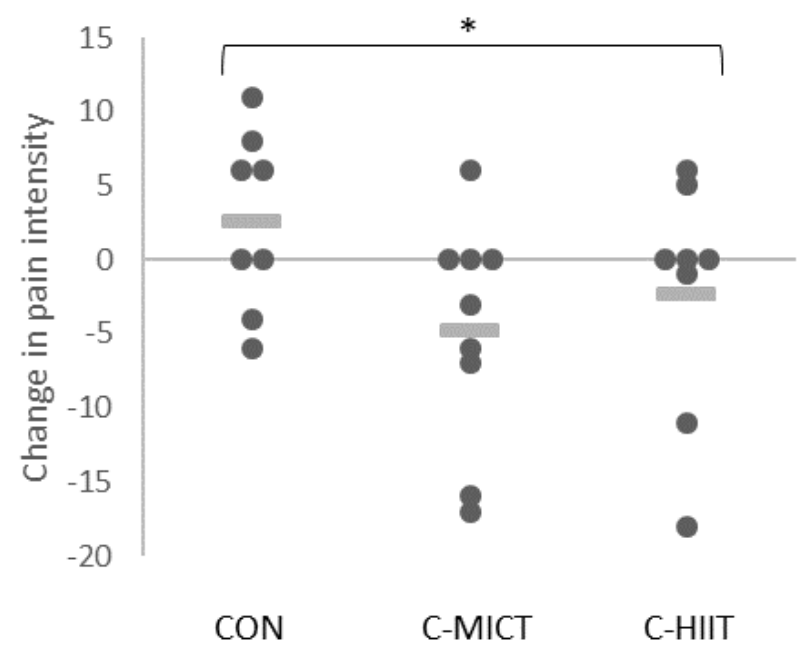

Figure 2a: Change in global pain intensity over the past week (evaluated by sum of numerical pain ratings across nine regions) for individual participants (dots) and mean scores for each group (lines). Negative scores represent improvement in pain from baseline to post intervention. *Significant difference between CON and C-HIIT $(\mathrm{p}<0.05)$.

Changes in neuropathy symptoms from baseline to post-intervention for individual participants (and mean scores for each group) are illustrated in Figure $2 \mathrm{~b}$. No differences were observed between C-MICT and CON (MD 0.2, 95\% CI -3.1 to 3.6; $\mathrm{p}=0.89$ ), C-HIIT and CON (MD 1.0, $95 \% \mathrm{CI}-0.9$ to $2.8, \mathrm{p}=0.31$ ), or the pooled exercise group and CON (MD $0.9,95 \% \mathrm{CI}-1.8$. to $3.5, \mathrm{p}=0.53$ ). Results were unchanged when excluding participants not meeting the prescribed intensity for $80 \%$ of sessions.

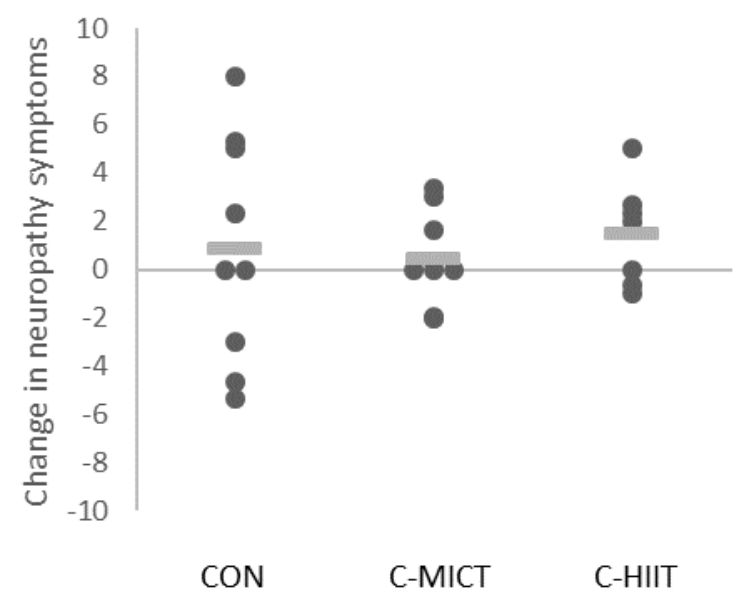

Figure 2b: Change in Neuropathy Total Symptom Score (NTSS-6) over the previous 24 hours for individual participants (dots) and mean scores for each group (lines). Negative scores represent improvement in symptoms from baseline to post intervention. 


\subsubsection{Effect of Exercise Training on Sensory Function}

No differences were observed between dominant and non-dominant limbs for sensory tests hence an average of both sides was used in analysis. No differences were observed between groups for sensory measures at baseline (all $\mathrm{p}>0.05$ ). No differences were also found at 8 weeks between C-HIIT and CON, or C-MICT and CON, for warm, cold, vibration or mechanical detection thresholds or pressure pain thresholds (all $\mathrm{p}>0.05$ ) (Supplementary Table 1).

\subsubsection{Adverse Events}

Thirty-six adverse events were documented during the training intervention (17 events in participants allocated to C-MICT and 19 events in participants allocated to C-HIIT). The number and type of events are listed in Table 2. Musculoskeletal adverse events were most prevalent $(72.2 \%$ of total events), followed by hypoglycaemia or dizziness $(27.8 \%$ of total events). The number of musculoskeletal events did not differ between C-MICT and C-HIIT, with $78 \%$ of all participants experiencing at least one musculoskeletal adverse event. Of the musculoskeletal adverse events, back pain accounted for $41.7 \%$ events in the C-HIIT group, but $0 \%$ of the C-MICT group, whereas pain in the lower limb accounted for in $8 \% \mathrm{C}$-HIIT, compared to $57.1 \%$ of C-MICT group. All events were classified as grade 1 (mild symptoms, intervention not indicated), except for one grade 2 event in the C-MICT group. The participant reported moderate shoulder pain during shoulder press exercise requiring cessation of the exercise, though this had no impact on their ability to exercise in following training sessions.

Table 2: Adverse events documented during training intervention according to Common Terminology Criteria for Adverse Events criteria.

\begin{tabular}{lll} 
Adverse event & C-MICT & C-HIIT \\
\hline Total events & $\mathbf{1 7}$ & $\mathbf{1 9}$ \\
Hypoglycaemia & 3 & 5 \\
Dizziness & 0 & 2 \\
Back pain & 0 & 5 \\
Decreased lumbar spine range of motion & 1 & 0 \\
Buttock pain & 2 & 0 \\
Pain in knee & 3 & 0 \\
Pain in leg & 2 & 0 \\
Pain in foot & 3 & 1 \\
Pain in shoulder & $1^{\mathrm{a}}$ & 3 \\
Myalgia & 2 & 3 \\
Risk of adverse events per 100 hours of training & 7 & 23 \\
Risk of musculoskeletal adverse events per 100 hours of & 6 & 14 \\
training & &
\end{tabular}

${ }^{a}$ Grade 2 adverse event 


\subsection{Discussion}

This exploratory study provides preliminary evidence supporting the efficacy and safety of low-volume combined aerobic and resistance high-intensity interval training (C-HIIT) for reducing musculoskeletal pain in inactive individuals with type 2 diabetes. Using a global pain intensity score, we identified a greater reduction in global pain intensity in participants allocated to 8-weeks of C-HIIT compared to usual care (CON). Similar reductions in global pain intensity were seen for participants allocated to combined moderate-intensity aerobic and resistance training (C-MICT) compared to $\mathrm{CON}$, although these were not statistically significant. When excluding participants who did not meet the prescribed intensity for $80 \%$ of the sessions, both exercise groups showed a significant reduction in global pain intensity compared to CON. No effect of exercise training on self-reported neuropathy symptoms or sensory function was detected.

Few RCTs have specifically examined the effects of exercise training on chronic pain in people with type 2 diabetes. In a systematic review of 136 RCTs investigating interventions to improve physical activity levels in people with diabetes, only one reported chronic pain measures at baseline and no trials investigated chronic pain as a target of the intervention [30]. Since this review, the Italian Diabetes and Exercise study published data from their RCT of 606 inactive people with type 2 diabetes comparing twice weekly supervised aerobic and resistance training plus counselling versus counselling alone for 12 months [11]. In a subgroup of 120 participants, lower (better) joint specific musculoskeletal scores (Roland-Morris, Constant Murley and Harris Hip questionnaires) were observed after exercise and counselling compared to counselling only. The authors proposed that the beneficial effect of exercise might be intensity dependent, as participants who performed higher intensity of exercise during the supervised sessions had better joint specific musculoskeletal scores. Unfortunately, scores were not evaluated at baseline for their cohort. Munk Jensen and colleagues (2019) recently found 12weeks of combined aerobic and resistance exercise training reduced musculoskeletal pain, as measured by a self-report $0-10$ Numeric Rating Scale, in people with type 2 diabetes, though they had no control group for comparison [31].

A potential explanation for the analgesic effects of higher intensity exercise is that the demanding nature of the exercise leads to the release of endogenous opioids [12]. Previous studies in healthy participants support the intensity-response analgesic effects of exercise. For example, a greater analgesic effect was induced by vigorous intensity aerobic exercise $(70 \%$ of heart rate reserve [HRR]) compared with moderate intensity aerobic exercise (50\% HRR) [32]. Additionally, a single session of HIIT ( 4 x 4 minutes at $85 \%$ HRR) induced analgesia in healthy, untrained young males [12]. Differential acute effects of HIIT and MICT on inflammatory (IL-6) function have also been observed in obese individuals [33]. Alternative mechanisms for the observed reduction in pain following exercise training include changes in physical function, body composition, and/or psychological/social factors (e.g. less fear or distress, and increased confidence in physical activity despite persisting pain) [34]. Larger studies are needed to further evaluate whether the effects of exercise on musculoskeletal pain are dose-dependent. 
Our finding of no statistically significant change in neuropathic symptoms is supported by some, but not all, research. Kluding and colleagues (2012) examined the effect of a 10-week program including aerobic (50-75\% $\dot{\mathrm{V}} \mathrm{O}_{2}$ reserve) and strengthening (RPE 7-8/10) exercise on pain in 17 adults with diabetic polyneuropathy [35]. Participants who completed at least $75 \%$ of the sessions experienced significant reductions in pain and neuropathic symptoms from baseline to post-intervention as measured by visual analogue scales and the Michigan Neuropathy Screening instrument. These findings were corroborated by Dixit, Maiya \& Shastry (2014) who found reductions in neuropathic symptoms, as measured by the Michigan Neuropathy Screening instrument, and improvements in quality of life, as measured by the Neuropathy Quality of Life instrument, in 29 individuals with diabetic peripheral neuropathy following 8-weeks of moderate intensity aerobic (40-60\% HRR) exercise training compared to control. In contrast, Yoo and colleagues (2015) examined a 16-week aerobic exercise intervention (50-75\% $\dot{\mathrm{VO}}_{2}$ reserve) in 14 inactive adults with painful peripheral neuropathy [36]. They found the program reduced perceived interference of pain on daily activities, but had no impact on pain severity. Importantly, only one of these small studies included a control group, so there is currently limited evidence as to whether exercise can alleviate pain in people with diabetic polyneuropathy. A potential explanation for the lack of change in neuropathic symptoms may be that neuropathic pain interferes with a person's willingness to participate in an exercise program $[34,37]$, or that neuropathic pain may interfere with pain modulation during exercise [38]. Participants in our training intervention that were lost to follow-up had higher baseline neuropathy symptoms than those who completed the intervention. Given this and the fact that our cohort included participants without symptoms of neuropathy, a floor effect may be present. As long-term supervised exercise has been found to delay (or prevent) the onset of diabetic polyneuropathy in people with type 2 diabetes [39], we also cannot discount that a longer training period may result in changes in the intensity or frequency of neuropathic symptoms.

The strengths of the current study include the high adherence to training intensities, which suggests that both supervised C-MICT and C-HIIT are feasible in inactive individuals with type 2 diabetes. While three out of four participants in both training interventions experienced at least one musculoskeletal adverse event, these were almost exclusively classified as grade 1 (minor symptoms not requiring treatment and had no impact on exercise training). Similar rates have been reported in other training studies in participants with diabetic neuropathy [40]. The total number of musculoskeletal adverse events was similar for C-MICT and C-HIIT. However, when adjusted for the number of training hours completed, the C-HIIT group had twice as many musculoskeletal adverse events. Therefore, although C-HIIT resulted in significant reductions in pain intensity compared to $\mathrm{CON}$, there may be a greater risk of minor adverse events with this type of training. Furthermore, interestingly, we identified potentially different distributions of musculoskeletal complaints, with more frequent reports of back pain in participants undergoing C-HIIT and more common lower extremity symptoms in participants undergoing C-MICT. It is plausible that the higher speed requirements of HIIT may cause greater strain on the spine, hence pose risk of back injury or exacerbation. Higher cumulative load on the lower extremity in participants undergoing C-MICT may increase the risk of leg 
and foot pain, especially plantar heel pain. Delayed onset muscle soreness was also common in both exercise groups.

Limitations of the study include the small sample size and exploratory investigation of the effects of exercise training on self-reported symptoms. A lack of validated instruments exist to capture the multidimensional and multisite nature of symptoms experienced by people with type 2 diabetes [2]. As widespread chronic pain is common in people with type 2 diabetes, we summed pain intensity scores across nine areas of the body as an index of global pain intensity. However, only $72 \%$ of participants reported musculoskeletal trouble in the last week, hence floor effects were likely observed. More importantly, the findings should be interpreted with caution as the clinical importance of these statistical differences is not known. Future studies should consider whether the small degree of improvement observed is important or trivial from the patient's perspective [41]. In contrast, we used a validated measure of neuropathic pain symptoms, alongside quantitative sensory tests, suggesting the exercise training did not change neuropathic symptoms or sensory function. Finally, this was a supervised intervention and the training program was developed and monitored by an Exercise Specialist. Therefore, conclusions cannot be drawn about the safety and feasibility of high-intensity exercise in an unsupervised setting in this population

\subsection{Conclusion}

These findings are relevant to the prescription of exercise for inactive individuals with type 2 diabetes and musculoskeletal pain. The current preliminary evidence suggests potential small benefits of low-volume, high-intensity combined aerobic and resistance exercise training on musculoskeletal pain after only 8-weeks. However, the evidence suggests that exercise may not be a panacea for neuropathic pain. Exercise Specialists may need to provide close monitoring and education to address anticipated minor adverse events related to musculoskeletal pain as people with type 2 diabetes begin an exercise program. As an exploratory study of relatively small sample size, these conclusions should be interpreted with caution until replicated in a larger trial.

\section{Acknowledgments}

The authors would like to acknowledge Diabetes Queensland for their assistance with participant recruitment.

\section{Data availability}

Data are available on request from the authors. 


\section{Funding}

This research received no specific grant from any funding agency in the public, commercial or not-for-profit sectors. BK Coombes was in receipt of a University of Queensland Research Fellowship for Women.

\section{Duality of Interest}

No conflicts of interest were present.

\section{Contributing Statement}

All authors contributed to the conception and design of the study, acquisition of data, or analysis/interpretation of data. All authors contributed to revising and final approval of the manuscript. BK Coombes accepts full responsibility for the work and or conduct of the study, had access to the data and controlled the decision to publish. 


\section{References}

[1] Cassidy, S., C. Thoma, D. Houghton, and M.I. Trenell, High-intensity interval training: A review of its impact on glucose control and cardiometabolic health. Diabetologia, 2017. 60(1): 7-23 10.1007/s00125-016-4106-1.

[2] Cox, E.R., J.S. Coombes, S.E. Keating, N.W. Burton, and B.K. Coombes, Not a painless condition: Rheumatological and musculoskeletal symptoms in type 2 diabetes, and the implications for exercise participation. Current Diabetes Reviews, 2019. 15 http://dx.doi.org/10.2174/1573399815666190531083504.

[3] Mantyselka, P., J. Miettola, L. Niskanen, and E. Kumpusalo, Persistent pain at multiple sites--connection to glucose derangement. Diabetes Res Clin Pract, 2009. 84(2): e30-2 10.1016/j.diabres.2009.01.018.

[4] Molsted, S., J. Tribler, and O. Snorgaard, Musculoskeletal pain in patients with type 2 diabetes. Diabetes Res Clin Pract, 2012. 96(2): 135-40 http://dx.doi.org/10.1016/j.diabres.2011.12.022.

[5] Abaraogu, U.O., C. Ochi, E. Umahi, C. Ogbonnaya, and I. Onah, Individuals with type 2 diabetes are at higher risk of chronic musculoskeletal pain: A study with diabetes cohort. Int J Diabetes Dec Ctries, 2017. 37(3): 267-71 10.1007/s13410-016-0489-2.

[6] van der Zee-Neuen, A., P. Putrik, S. Ramiro, A. Keszei, R. de Bie, A. Chorus, et al., Impact of chronic diseases and multimorbidity on health and health care costs: The additional role of musculoskeletal disorders. Arthritis Care Res (Hoboken), 2016. 68(12): 1823-31 10.1002/acr.22913.

[7] Buford, T.W., M.D. Roberts, and T.S. Church, Toward exercise as personalized medicine. Sports Med, 2013. 43(3): 157-65 10.1007/s40279-013-0018-0.

[8] Liu, J.X., L. Zhu, P.J. Li, N. Li, and Y.B. Xu, Effectiveness of high-intensity interval training on glycemic control and cardiorespiratory fitness in patients with type 2 diabetes: A systematic review and meta-analysis. Aging Clin Exp Res, 2018; 10.1007/s40520-018-1012-z.

[9] Weston, K.S., U. Wisloff, and J.S. Coombes, High-intensity interval training in patients with lifestyle-induced cardiometabolic disease: A systematic review and metaanalysis. Br J Sports Med, 2014. 48(16): 1227-34 10.1136/bjsports-2013-092576.

[10] Jung, M.E., J.E. Bourne, and J.P. Little, Where does hit fit? An examination of the affective response to high-intensity intervals in comparison to continuous moderateand continuous vigorous-intensity exercise in the exercise intensity-affect continuum. PLoS One, 2014. 9(12): e114541 10.1371/journal.pone.0114541.

[11] Balducci, S., M.C. Vulpiani, L. Pugliese, V. D'Errico, S. Menini, G. Salerno, et al., Effect of supervised exercise training on musculoskeletal symptoms and function in patients with type 2 diabetes: The italian diabetes exercise study (ides). Acta Diabetol, 2014. 51(4): 647-54 10.1007/s00592-014-0571-5.

[12] Kodesh, E. and I. Weissman-Fogel, Exercise-induced hypoalgesia - interval versus continuous mode. Appl Physiol Nutr Metab, 2014. 39(7): 829-34 10.1139/apnm2013-0481.

[13] Johansen, M.Y., C.S. MacDonald, K.B. Hansen, K. Karstoft, R. Christensen, M. Pedersen, et al., Effect of an intensive lifestyle intervention on glycemic control in patients with type 2 diabetes: A randomized clinical trial. JAMA, 2017. 318(7): 637-46 10.1001/jama.2017.10169. 
[14] Praet, S.F., E.S. van Rooij, A. Wijtvliet, L.J. Boonman-de Winter, T. Enneking, H. Kuipers, et al., Brisk walking compared with an individualised medical fitness programme for patients with type 2 diabetes: A randomised controlled trial. Diabetologia, 2008. 51(5): 736-46 10.1007/s00125-008-0950-y.

[15] Hordern, M.D., D.W. Dunstan, J.B. Prins, M.K. Baker, M.A. Singh, and J.S. Coombes, Exercise prescription for patients with type 2 diabetes and pre-diabetes: A position statement from exercise and sport science australia. J Sci Med Sport, 2012. 15(1): 2531 10.1016/j.jsams.2011.04.005.

[16] Colberg, S.R., A.L. Albright, B.J. Blissmer, B. Braun, L. Chasan-Taber, B. Fernhall, et al., Exercise and type 2 diabetes: American college of sports medicine and the american diabetes association: Joint position statement. Exercise and type 2 diabetes. Med Sci Sports Exerc, 2010. 42(12): 2282-303.

[17] Oliveira, C., M. Simoes, J. Carvalho, and J. Ribeiro, Combined exercise for people with type 2 diabetes mellitus: A systematic review. Diabetes Res Clin Pract, 2012. 98(2): 187-98 10.1016/j.diabres.2012.08.004.

[18] Christ-Roberts, C.Y., T. Pratipanawatr, W. Pratipanawatr, R. Berria, R. Belfort, S. Kashyap, et al., Exercise training increases glycogen synthase activity and glut4 expression but not insulin signaling in overweight nondiabetic and type 2 diabetic subjects. Metabolism, 2004. 53(9): 1233-42.

[19] Praet, S.F., R.A. Jonkers, G. Schep, C.D. Stehouwer, H. Kuipers, H.A. Keizer, et al., Long-standing, insulin-treated type 2 diabetes patients with complications respond well to short-term resistance and interval exercise training. Eur J Endocrinol, 2008. 158(2): 163-72 10.1530/eje-07-0169.

[20] Francois, M.E., C. Durrer, K.J. Pistawka, F.A. Halperin, C. Chang, and J.P. Little, Combined interval training and post-exercise nutrition in type 2 diabetes: $A$ randomized control trial. Frontiers in Physiology, 2017. 8(528) 10.3389/fphys.2017.00528.

[21] De Feyter, H.M., S.F. Praet, N.M. van den Broek, H. Kuipers, C.D. Stehouwer, K. Nicolay, et al., Exercise training improves glycemic control in long-standing insulintreated type 2 diabetic patients. Diabetes Care, 2007. 30(10): 2511-3 10.2337/dc070183.

[22] Pescatello, L.S., R. Arena, D. Riebe, and P.D. Thompson, Acsm's guidelines for exercise testing and prescription. 9th ed, ed. A.C.o.S. Medicine. 2014, Philadelphia, PA: Wolters Kluwer/Lippincott Williams \& Wilkins. 456.

[23] Kuorinka, I., B. Jonsson, A. Kilbom, H. Vinterberg, F. Biering-Sorensen, G. Andersson, et al., Standardised nordic questionnaires for the analysis of musculoskeletal symptoms. Appl Ergon, 1987. 18(3): 233-7.

[24] Dickinson, C.E., K. Campion, A.F. Foster, S.J. Newman, A.M. O'Rourke, and P.G. Thomas, Questionnaire development: An examination of the nordic musculoskeletal questionnaire. Appl Ergon, 1992. 23(3): 197-201.

[25] Bastyr, E.J., 3rd, K.L. Price, and V. Bril, Development and validity testing of the neuropathy total symptom score-6: Questionnaire for the study of sensory symptoms of diabetic peripheral neuropathy. Clin Ther, 2005. 27(8): 1278-94 10.1016/j.clinthera.2005.08.002.

[26] U.S. Department of Health and Human Services, Common terminology criteria for adverse events (ctcae) v5.0. 2017: Maryland (USA). 
[27] Craig, C.L., A.L. Marshall, M. Sjostrom, A.E. Bauman, M.L. Booth, B.E. Ainsworth, et al., International physical activity questionnaire: 12-country reliability and validity. Med Sci Sports Exerc, 2003. 35(8): 1381-95 10.1249/01.Mss.0000078924.61453.Fb.

[28] Wanner, M., N. Probst-Hensch, S. Kriemler, F. Meier, C. Autenrieth, and B.W. Martin, Validation of the long international physical activity questionnaire: Influence of age and language region. Preventive medicine reports, 2016. 3: 250-6 10.1016/j.pmedr.2016.03.003.

[29] Borg, G., An introduction to borg's rpe scale. 1985, Ithaca, N.Y.: Mouvement Publications.

[30] Riva, J.J., J.J. Wong, D.J. Brunarski, A.H. Chan, R.A. Lobo, M. Aptekman, et al., Consideration of chronic pain in trials to promote physical activity for diabetes: A systematic review of randomized controlled trials. PLoS One, 2013. 8(8): e71021 10.1371/journal.pone.0071021.

[31] Munk Jensen, T., S. Bjerre Milling Eriksen, J. Sedum Larsen, M. Aadahl, S. Sætre Rasmussen, L. Bockhoff Olesen, et al., Exercise training is associated with reduced pains from the musculoskeletal system in patients with type 2 diabetes. Diabetes Research and Clinical Practice, 2019. 154: 124-9 https://doi.org/10.1016/j.diabres.2019.07.003.

[32] Naugle, K.M., K.E. Naugle, R.B. Fillingim, B. Samuels, and J.L. Riley, 3rd, Intensity thresholds for aerobic exercise-induced hypoalgesia. Med Sci Sports Exerc, 2014. 46(4): 817-25 10.1249/MSS.0000000000000143.

[33] Barry, J.C., S. Simtchouk, C. Durrer, M.E. Jung, and J.P. Little, Short-term exercise training alters leukocyte chemokine receptors in obese adults. Med Sci Sports Exerc, 2017. 49(8): 1631-40 10.1249/MSS.0000000000001261.

[34] Geelen, C.C., R. Smeets, S. Schmitz, J.P. van den Bergh, M. Goossens, and J.A. Verbunt, Anxiety affects disability and quality of life in patients with painful diabetic neuropathy. Eur J Pain, 2017. 21(10): 1632-41 10.1002/ejp.1067.

[35] Kluding, P.M., M. Pasnoor, R. Singh, S. Jernigan, K. Farmer, J. Rucker, et al., The effect of exercise on neuropathic symptoms, nerve function, and cutaneous innervation in people with diabetic peripheral neuropathy. J Diabetes Complications, 2012. 26(5): 424-9 10.1016/j.jdiacomp.2012.05.007.

[36] Yoo, M., L.J. D'Silva, K. Martin, N.K. Sharma, M. Pasnoor, J.W. LeMaster, et al., Pilot study of exercise therapy on painful diabetic peripheral neuropathy. Pain Med, 2015. 16(8): 1482-9 10.1111/pme.12743.

[37] Geneen, L.J., R.A. Moore, C. Clarke, D. Martin, L.A. Colvin, and B.H. Smith, Physical activity and exercise for chronic pain in adults: An overview of cochrane reviews. Cochrane Database Syst Rev, 2017;(4): CD011279 10.1002/14651858.CD011279.pub3.

[38] Knauf, M.T. and K.F. Koltyn, Exercise-induced modulation of pain in adults with and without painful diabetic neuropathy. J Pain, 2014. 15(6): 656-63 10.1016/j.jpain.2014.02.008.

[39] Balducci, S., G. lacobellis, L. Parisi, N. Di Biase, E. Calandriello, F. Leonetti, et al., Exercise training can modify the natural history of diabetic peripheral neuropathy. J Diabetes Complications, 2006. 20(4): 216-23 10.1016/j.jdiacomp.2005.07.005.

[40] Kluding, P.M., M. Pasnoor, R. Singh, L.J. D'Silva, M. Yoo, S.A. Billinger, et al., Safety of aerobic exercise in people with diabetic peripheral neuropathy: Single-group clinical trial. Phys Ther, 2015. 95(2): 223-34 10.2522/ptj.20140108. 
[41] Redelmeier, D.A. and K. Lorig, Assessing the clinical importance of symptomatic improvements. An illustration in rheumatology. Arch Intern Med, 1993. 153(11): 1337-42. 